\title{
Naussac 2 : mise en œuvre des pompes turbines Deriaz
}

\author{
Naussac 2 : using turbine pumps Deriaz
}

\author{
par R. Thépot \\ Epala \\ J. Clérin, C. Sudour \\ Somival \\ J.P. Taulan \\ Alstom Hydro Turbines Neyrpic
}

EPALA, the owner, has entrusted Somival, the project manager, and Neyrpic with the construction of the hydroelectric part of the second phase of the Naussac installation, ensuring additional reservoir filling by pumping. The choice of Deriaz pump-turbines was fundamental to obtain the flexibility required for using the Allier water ressources. These machines are able to operate over a relatively wide range of flows and upstream loads and so adapt to the site's variable hydrological conditions.

The owner wanted to maintain the best possible quality of pumped water, which meant installing " green" machines with a number of innovations.

\section{L'AMÉNAGEMENT DE NAUSSAC}

\subsection{Caractéristiques de l'aménagement}

L'aménagement de NAUSSAC près de Langogne (Lozère), sur le haut bassin de l'Allier, est destiné au soutien d'étiage de l'Allier et de la Loire. Il a été prévu en deux phases de réalisation en 1973 .

La première phase, mise en service en 1983, a permis de disposer d'un réservoir de $190 \mathrm{Mm}^{3}$ de capacité par création d'un barrage sur le Donozau. Ce réservoir est alimenté par le bassin versant propre du Donozau $\left(20 \mathrm{Mm}^{3}\right.$ en apports moyens annuels) et le bassin dérivé du Chapeauroux, au moyen d'une galerie de $2 \mathrm{~km}$ (60 à $70 \mathrm{Mm}^{3}$ en apports moyens annuels). Cette phase a été réalisée par Somival, concessionnaire de l'Etat.

La deuxième phase, réalisée par l'EPALA, et dont la mâ̂trise d'œuvre a été confiée à Somival, consiste à compléter le remplissage du réservoir par pompage des eaux de l'Allier. Elle a nécessité la réalisation d'un ouvrage de prise sur l'Allier avec transfert des eaux au pied aval du barrage où a été établi un bassin de pompage (voir figure 1).

Une usine en puits creusée au pied immédiat du barrage accueille trois machines identiques de type Deriaz, de $3 \mathrm{MW}$ chacune, à débit et à charge variables, de conception et réalisation Alstom Hydro Turbines Neyrpic ${ }^{\circledR 3}$ sur brevet GEC (figure 2).
En fonction d'une courbe d'alerte basée sur une garantie à $90 \%$ de remplissage total de la retenue au $1^{\text {er }}$ juin et en fonction des débits de l'Allier, les pompes sont mises en marche.

Les machines sont réversibles, permettant ainsi de turbiner les lâchures de soutien d'étiage et donc de réduire les coûts de fonctionnement de l'aménagement.

Les particularités de la deuxième phase d'aménagement résident essentiellement dans :

- le choix du type de machine,

- le fonctionnement sans huile de certains organes.

\subsection{Choix des machines DERIAZ}

La particularité des Deriaz est leur souplesse d'utilisation et leur plage étendue de fonctionnement, quelle que soit la charge amont. Le débit unitaire de pompage est ainsi variable de 1 à $5,5 \mathrm{~m}^{3} / \mathrm{s}$ sous une charge variant de 32 à $57 \mathrm{~m}$.

C'est cette souplesse de fonctionnement qui a séduit I'EPALA. Le choix de ces machines innovantes a été fait par le Maître d'Ouvrage dans le souci d'intégrer le mieux possible la centrale dans l'environnement. Elles permettent en effet d'ajuster en permanence, " au fil de l'eau ", le débit pompé à celui apporté par la rivière, de façon à éviter de brusques marnages dans l'Allier. Cela permet de réduire aussi au strict minimum le volume du bassin de pompage, et de se contenter d'un petit seuil à clapet sur le Donozau. 


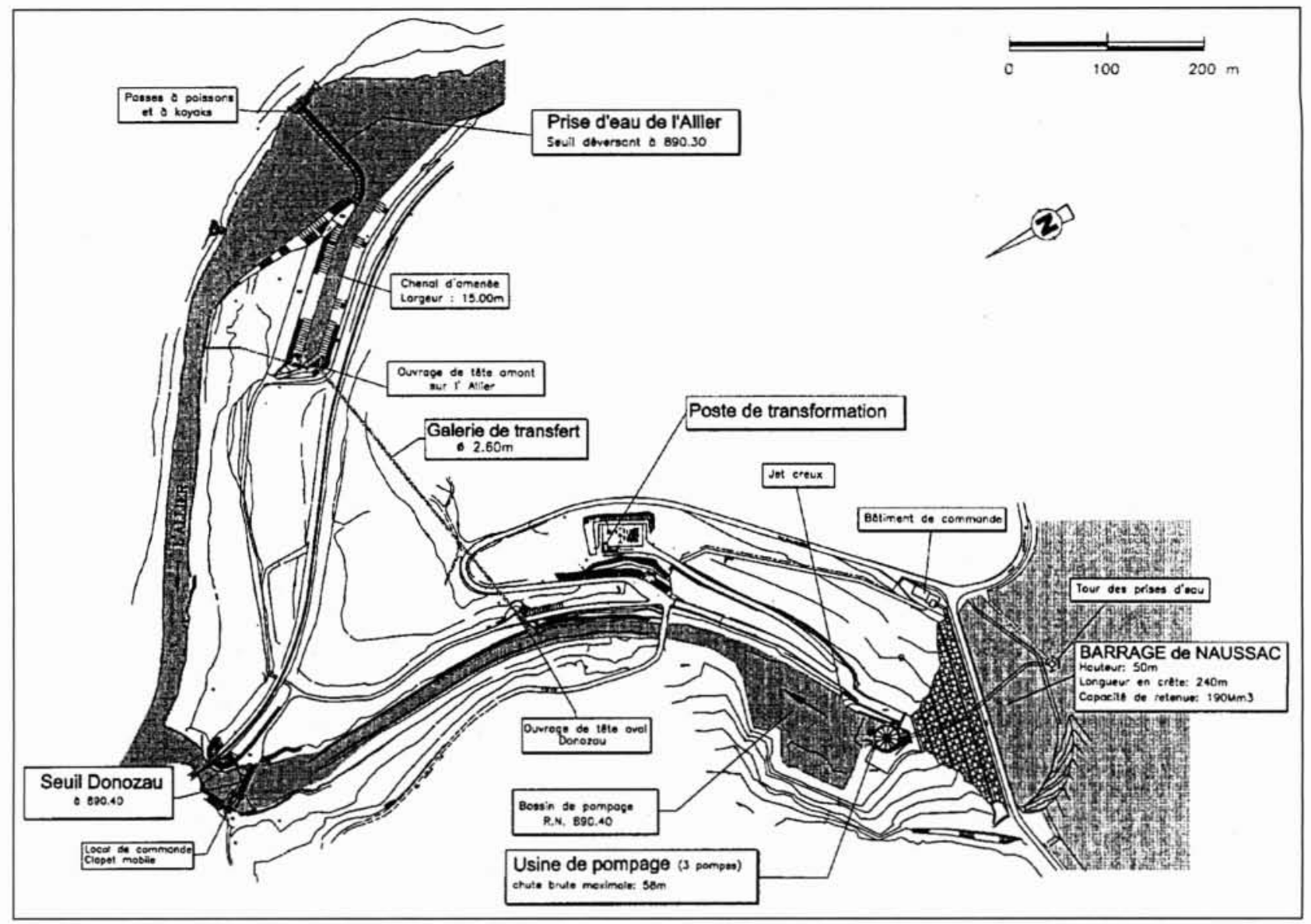

1. Plan général de l’aménagement.

Les solutions techniques alternatives, avec des machines type Francis bivitesses ou multivitesses, auraient nécessité un bassin de pompage (ou de compensation) de capacité bien supérieure. Il en aurait découlé un ouvrage de prise sur l'Allier de plus de $10 \mathrm{~m}$ de hauteur alors qu'il n'est que de 2,30 m grâce aux machines Deriaz, ce qui est beaucoup plus favorable tant pour le milieu que pour l'intégration paysagère.

Cette réalisation avec des Deriaz est une première européenne qui lui a valu d'obtenir un soutien financier de la Communauté Européenne au titre de projet de démonstration et projet pilote industriel dans le domaine de l'énergie.

\section{- 1.3 Fonctionnement " sans huile"}

La prise en compte des problèmes de qualité d'eau a conduit le maître d'ouvrage à retenir des dispositifs techniques permettant de qualifier les machines tournantes mises en place de " vertes" . Les trois machines sont en effet conçues pour éviter les risques de pollution de l'environnement, grâce à des technologies résolument modernes : le palier-joint hydrostatique et les organes de commande des pales de la roue sont revêtus de céramique et fonctionnent à l'eau, de même que les robinets de garde et les vannes-aval. D'autres organes comme le vannage des pompes sont à commande électrique. C'est la première fois que ces dispositifs innovants ont été mis en oeuvre par Neyrpic conjointement sur une même machine. Ils excluent l'utilisation de fluide polluant tel que l'huile dans les parties de la machine pouvant être en contact avec l'eau pompée ou turbinée. Quelques détails de leur mise en œuvre et des difficultés rencontrées sont donnés dans les chapitres suivants.
Les turbomachines " vertes " réversibles Deriaz répondent ainsi parfaitement aux souhaits de limitation des variations brusques de débit et de niveaux en s'adaptant aux contraintes hydrologiques. Leur insertion paysagère a pu se faire sans grande perturbation de l'environnement, et leur fonctionnement " à l'eau " élimine quasiment tout risque de pollution.

\subsection{Historique de la mise en æuvre des pompes-tur-} bines Deriaz à Naussac

Les travaux sur site ont été réalisés de mars 1995 à décembre 1997. Les premiers tours de roue, en commençant par le fonctionnement en turbine, ont eu lieu en novembre 1997. Les trois machines avaient été essayées en pompe en décembre 1997.

La mise au point délicate de certains organes innovants a retardé de quelques mois la mise en service industrielle des machines, effectuée au printemps 1998. Les principales difficultés survenues sont mentionnées dans le troisième chapitre de la présente publication.

\section{II $\square$ MISE EN GEUVRE DES POMPES- TURBINES DERIAZ}

\subsection{Caractéristiques générales}

Les trois groupes hydroélectriques réversibles identiques de la centrale de Naussac 2 ont une puissance unitaire de $3000 \mathrm{~kW}$ en pompe et de $2650 \mathrm{~kW}$ en turbine. Chaque groupe est accouplé à un moteur-générateur asynchrone clas- 


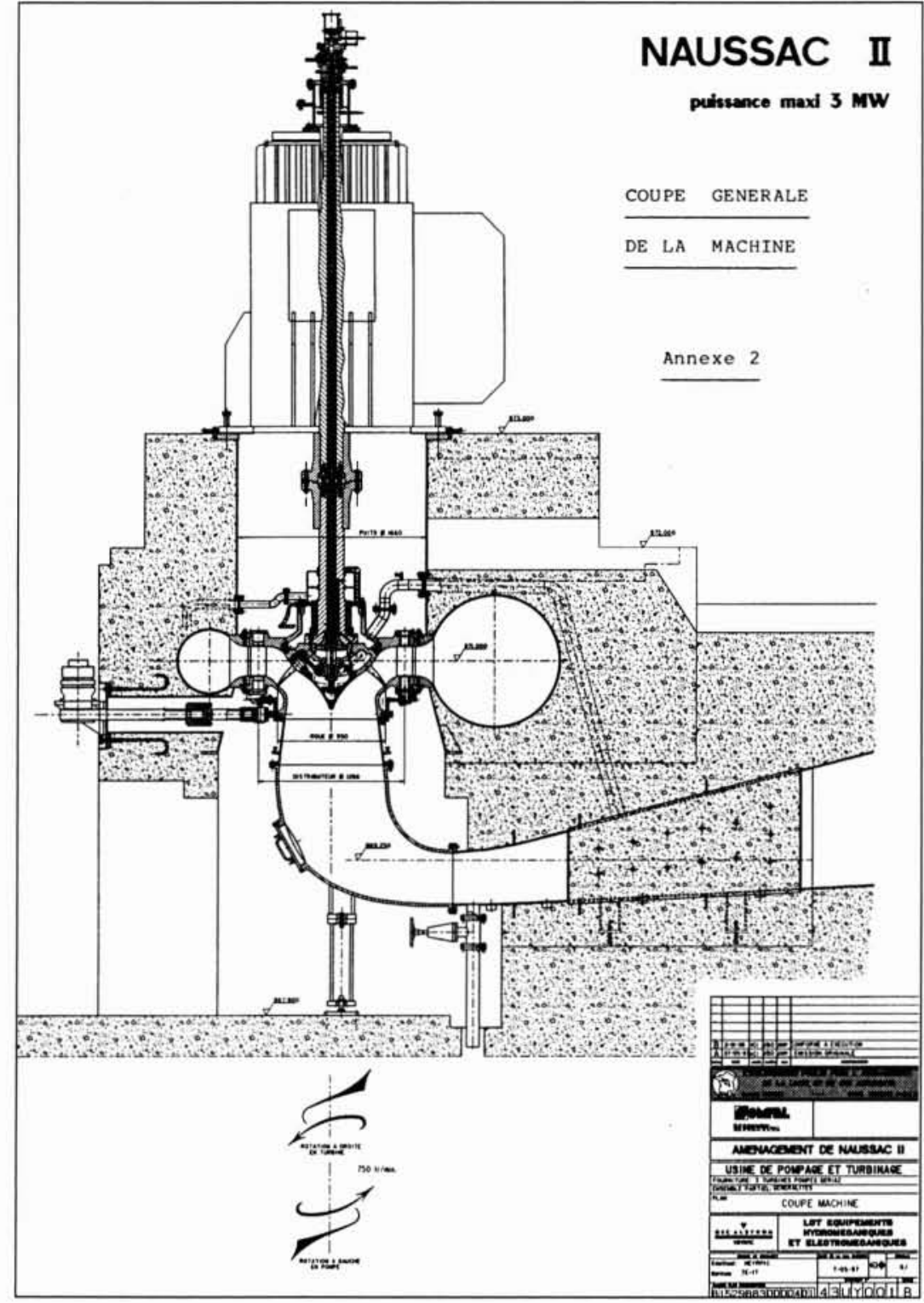

2. Coupe générale de la machine de type Deriaz.

Une seule centrale hydraulique par groupe est utilisée, commune au robinet et à la vanne aval.

Les trois aspirateurs des groupes se rassemblent dans un puits commun d'aspiration à l'aval de l'usine. Celui-ci comporte également grilles et batardeaux.

Afin d'éviter que l'aspirateur d'un groupe ne soit accidentellement mis à la pression amont, le fonctionnement de la vanne aval est étroitement lié à celui du robinet papillon de garde, la fermeture de celle-ci n'étant autorisée qu'après détection de la fermeture totale de ce dernier. En cas de fuite sur le robinet alors que la vanne est fermée, l'augmentation de pression dans l'aspirateur repousse le tablier vers l'aval. Le jeu ainsi créé entre les joints d'étanchéité de la vanne et les pièces fixes est alors suffisant pour évacuer le débit de fuite sans que la différence de pression entre les deux côtés de la vanne ne dépasse 5 mètres de colonne d'eau.

\subsection{Caractéristiques hydrauliques}

Deriaz, c'est le nom de l'inventeur de ce type de pompe-turbine spécifique. II s'agit d'une turbomachine réversible à flux diagonal, avec distributeur réglable et pales orientables, capable de régler son débit et de s'adapter aux grandes variations de chute, grâce à ses pales mobiles. La principale caractéristique de celles-ci, les distinguant de celles d'une turbine classique de type Kaplan, est d'avoir leurs axes inclinés comme les baleines d'un parapluie et non dans un plan horizontal. Elles ont ainsi un profil hydraulique leur permettant de travailler, aussi bien en turbine qu'en pompe, sous des chutes ou des charges amont relativement plus élevées qu'avec une Kaplan.

Les caractéristiques de fonctionnement

sique. L'ensemble des trois groupes alimente un transformateur unique de $5.5 \mathrm{kV} / 63 \mathrm{kV}$ de $12 \mathrm{MVA}$.

Une seule conduite regroupe les débits des trois machines au moyen d'une trifurcation située au pied aval du barrage, à l'amont immédiat du puits de l'usine. Le raccordement à la conduite existante sous le barrage est effectué par l'intermédiaire d'un répartiteur cylindrique réalisant le changement de direction à $90^{\circ}$ de l'écoulement. Cette structure originale comporte des grilles empêchant l'introduction de corps étrangers dans les machines lors du fonctionnement en turbine. Ces grilles ne disposent pas de dégrilleur mais peuvent être nettoyées à contre-courant au pompage, les détritus étant évacués par un conduit de chasse en dérivation. A l'amont, un batardeau permet d'isoler l'usine sans avoir à vidanger la totalité de la conduite jusqu'à la prise d'eau, et garantit donc le soutien d'étiage sans turbiner.

Chaque machine est dotée d'un robinet papillon à l'amont et d'une vanne wagon à l'aval. La commande de ces deux organes de garde est réalisée par des servomoteurs à eau. en pompe sont les suivantes

- puissance nominale absorbée par groupe

- débit correspondant par groupe de

- pour hauteur totale d'élévation de

$3 \mathrm{MW}$

- pertes de charge totales pour hauteur maxi
- correspondant à hauteur de chute brute

4,6 à $5,5 \mathrm{~m}^{3} / \mathrm{s}$ $58,2 \mathrm{~m}$ à $48,7 \mathrm{~m}$

$1,2 \mathrm{~m}$

- avec un nombre de groupes en service de

$57 \mathrm{~m}$

Les caractéristiques de fonctionnement en turbine sont les suivantes :

- puissance maximale utilisable par groupe

- débit correspondant par groupe de

$2,65 \mathrm{MW}$

pour hauteur de chute nette de

5,4 à $6,0 \mathrm{~m}^{3} / \mathrm{s}$

- pertes de charge totales pour hauteur maxi $\quad 0.5 \mathrm{~m}$

- correspondant à hauteur de chute brute $58 \mathrm{~m}$

- avec un nombre de groupe en service de

1

La conjugaison des pales et du vannage permet l'optimisation des performances des groupes et leur meilleure adapta- 
tion aux conditions de chute et de débit. Mais, elle n'empêche pas l'existence d'un feston, dans la caractéristique $H(Q)$ en pompe, comme on le trouve sur toute machine classique. Une loi de démarrage spécifique permet de bien passer le feston lors de la prise de charge. De même sur une baisse de charge, le régulateur interdit l'entrée dans le feston. Toutefois, des essais de fonctionnement effectués dans le feston ont montré que la pompe n'était ni particulièrement bruyante, ni instable dans cette partie de la caractéristique. L'inconvénient d'un pompage dans cette zone, s'il était autorisé, serait un rendement dégradé n'autorisant qu'un très faible débit de pompage comparé à la puissance absorbée par l'alternateur-moteur.

En fonctionnement normal, la machine est relativement bruyante jusqu'à des débits d'environ $3,5 \mathrm{~m}^{3} / \mathrm{s}$, puis devient peu sonore au-delà. Il s'agit d'un bruit de cavitation. Cette cavitation, déjà mise en évidence lors des essais sur modèle, a été jugée tout à fait acceptable à condition que les pales de la roue soient réalisées en matériau inoxydable. C'est le choix qui a été fait et qui permettra un fonctionnement satisfaisant sans dommage excessif. Le fonctionnement industriel à faibles débits permettra de confirmer cette analyse, mais il est préférable de limiter les périodes de pompage à trop faibles débits, ce que permet le bassin de pompage de $40000 \mathrm{~m}^{3}$ créé sur le Donozau. Les machines Deriaz ont beau être capables d'une grande souplesse d'adaptation, elles n'en restent pas moins, comme tout autre type de pompesturbines, mieux adaptées au point de meilleur rendement sous chaque chute, celui correspondant au débit nominal choisi.

\subsection{Caractéristiques mécaniques}

Les pompes-turbines de Naussac 2 ont des dimensions très modestes, adaptées à la valeur maximale de débit que le projet a défini comme utile de transférer de l'Allier vers le réservoir. Leur limitation de puissance en turbine a été fixée par la règle définissant en France la puissance maximale pouvant équiper une centrale hydroélectrique non gérée par EDF. Leurs principales caractéristiques mécaniques sont les suivantes :

- vitesse de synchronisme

$750 \mathrm{tr} / \mathrm{mn}$

- encombrement de la bâche spirale

$3178 \mathrm{~mm} \times 3666 \mathrm{~mm}$ en plan horizontal

- diamètre d'entrée de la bâche spirale

$1140 \mathrm{~mm}$

- nombre d'avant-directrices fixes

- nombre de directrices mobiles

- hauteur du distributeur à l'entrée

de roue (en turbine)

$257 \mathrm{~mm}$

- cote de l'axe du distributeur

- diamètre de perçage du distributeur

$871,0 \mathrm{NGF}$

- diamètre de la roue

$1286 \mathrm{~mm}$

- nombre de pales de la roue

$950 \mathrm{~mm}$

- largeur de la sortie de l'aspirateur

592

- hauteur de la sortie de l'aspirateur

$1130 \mathrm{~mm}$

- niveau du point bas de l'aspirateur

- cote à retenue normale du réservoir de NAUSSAC

$868,9 \mathrm{NGF}$

945,0 NGF

- cote à retenue normale du bassin de pompage 890,4 NGF

Les faibles dimensions de ces machines, loin de constituer une facilité de réalisation, ont plutôt contribué à compliquer davantage leur fabrication. Il n'est qu'à voir la hauteur de l'aspirateur pour comprendre toute la difficulté d'en construire la trompette mécano-soudée. Et quand on voit la roue, on a du mal à imaginer que puisse rentrer dans un aussi faible espace tout le mécanisme de commande des 8 pales.

A ces difficultés, inhérentes à la taille des machines, viennent s'ajouter celles liées à leur caractère de prototype. Ce sont les premières pompes-turbines Deriaz construites par Neyrpic. Le mécanisme de commande des pales avec servomoteur à eau intégré au moyeu de roue représente une prouesse technologique. De plus, le fonctionnement à l'eau de cette commande des pales est une première et a nécessité une adaptation du système classique des alvéoles avec revêtement de céramique sur les parties fixes et tournantes en contact. Enfin, le palier-joint hydrostatique principal de la pompe, conçu dans le même souci d'éviter toute pollution par l'huile de l'eau pompée ou turbinée, a été réalisé sur les mêmes bases que celui du groupe du Truel dans la centrale EDF du Pouget (Aveyron).

\subsection{Caractéristiques du contrôle-commande}

La régulation de vitesse de chaque groupe est réalisée par un régulateur DIGIPID 1500 Neyrpic. Les mesures de vitesse sont réalisées à partir de capteurs inductifs à oscillateur et d'une roue dentée. La détection de survitesse est faite par le régulateur à partir de l'information de vitesse ainsi fournie. Une détection du sens de rotation est réalisée à partir des indications de deux capteurs spécifiques. Dans la phase de démarrage en turbine, le régulateur amène puis maintient la machine au voisinage de la vitesse nominale synchrone permettant à celle-ci d'être couplée au réseau. Une fois le groupe couplé au réseau, le régulateur réalise l'asservissement du débit pompé ou turbiné à une consigne fournie par l'automatisme.

Le régulateur dispose de deux cames de conjugaison, une pour le fonctionnement en pompe, l'autre pour le fonctionnement en turbine, permettant d'assurer la meilleure conjugaison des pales et du vannage. Celle-ci correspond au meilleur rendement possible pour toutes les gammes de débits et de puissances sous toutes les hauteurs de refoulement en pompe et sous toutes les chutes en turbine.

L'aménagement de NAUSSAC est destiné au soutien d'étiage de l'Allier et de la Loire ; le fonctionnement des machines a donc été optimisé en pompe afin de privilégier le remplissage du réservoir, et non la production hydroélectrique qui est un sous-produit.

\section{MISE EN SERVICE DES MACHINES}

Des difficultés mineures ont été rencontrées lors de la mise en service des machines en décembre 1997 et en janvier 1998 :

- le 12 décembre 1997, incident au niveau des bagues d'alvéoles du groupe 2 ;

- le 9 janvier 1998, grippage du palier-joint hydrostatique du groupe 3.

Après analyse il est apparu que les deux incidents étaient dus à la détérioration du revêtement de céramique des bagues d'alvéoles et du palier. Il a été décidé d'appliquer un nouveau traitement de surface sur l'ensemble des pièces revêtues de céramique, y compris sur celles du groupe 1 qui n'avait pas subi d'incident. 


\section{- 3.1 Incident sur les bagues d'alvéoles du G2}

L'incident a été révélé par une augmentation anormale des débits de fuite des alvéoles, qui a entraîné une entrée d'eau intempestive dans le rotor du moteur de la pompe. Après démontage de la tête d'alvéoles, on a constaté des amorces de décollage du carbure de chrome pouvant être assimilées à un début de grippage. L'incident était lié principalement à un léger défaut de centrage des parties tournantes par rapport aux bagues fixes. Avec un bon revêtement de céramique, ce contact sec entre parties fixes et tournantes n'aurait pas dû entraîner les dégâts constatés. L'incident survenu ultérieurement sur le palier-joint du G3 a confirmé le manque de résistance au contact du revêtement d'origine, et a conduit à intervenir sur les trois niveaux suivants :

- modification de la nature du revêtement de céramique, avec adoption d'un oxyde de chrome en lieu et place du carbure de chrome d'origine,

- contrôle des coaxialités des tubes d'alvéoles. Il s'est avéré que certains usinages avaient été réalisés sur les manchons d'extrémité avant soudage de ceux-ci en bout de tubes. Deux nouveaux tubes ont été réalisés. Le remontage des tubes d'alvéoles et des bagues a été fait avec un contrôle plus rigoureux des concentricités,

- enfin, comme suite au grippage du palier principal de la pompe du G3, une analyse de toutes les pièces à fonctionnement hydrostatique a été entreprise, et a conduit à augmenter le nombre de poches d'alimentation de tous les paliers hydrostatiques. Un fonctionnement à 8 poches garantit dorénavant un autocentrage des bagues, même en cas d'obstruction de l'une d'elles.

\section{- 3.2 Grippage du palier-joint hydrostatique du G3}

Le grippage complet avec destruction des portées du palier et de l'arbre du groupe 3 est survenu sans qu'aucun signe précurseur n'ait pu être mis en évidence. Une signalisation d'un défaut d'arrosage a bien été relevée, mais après rupture du pressostat de contrôle du circuit d'alimentation, donc très probablement après incident sur le palier.

Plusieurs causes possibles ont pu concourir à cet incident : - mauvais comportement du revêtement de céramique d'origine, ayant entrainé la destruction du palier par endommagement initial des poches ; celles-ci ont été retrouvées colmatées par un amalgame de céramique ;

- mauvaise verticalité du coussinet entrainant un effet de coin et limitant considérablement l'effet hydrostatique du palier ;

- défaut d'arrosage furtif entraînant un déséquilibre de la ligne d'arbre et un contact destructeur au niveau du palier.

Ces trois causes possibles ont donc fait l'objet des trois traitements indépendants ci-dessous :

- remplacer le carbure de chrome, utilisé initialement sur recommandation de spécialistes, par de l'oxyde de chrome, déjà expérimenté sur le palier hydrostatique du TRUEL. Ce type de céramique est bien adapté aux paliers hydrostatiques, où certains contacts secs peuvent survenir lors de fonctionnements en régimes transitoires. Le carbure de chrome se comporte apparemment moins bien aux températures relativement basses, rencontrées dans les paliers hydrostatiques, qu'aux températures élevées où il est très largement expérimenté dans les paliers de turbines à gaz ou de réacteurs d'avion,
- réviser la procédure de montage de la ligne d'arbre utilisée précédemment, car elle n'excluait pas totalement un léger défaut d'alignement entre l'arbre et le coussinet de palier. La nouvelle procédure, mise au point avec des montages à blanc préliminaires, a permis un réglage précis du parallélisme entre arbre et coussinet, et un calage définitif des différentes parties mécaniques concernées,

- renforcer le dispositif d'arrosage des paliers hydrostatiques. Deux mesures simultanées ont été retenues pour améliorer le fonctionnement du palier-joint hydrostatique. La pression d'arrosage a été doublée pour augmenter la raideur du palier, et le nombre de poches d'alimentation a aussi été doublé, avec dédoublement du circuit et des capteurs correspondants.

Ces mesures ont été généralisées aux trois groupes.

\section{- 3.3 Mise en service industriel des machines}

Au cours du printemps 1998, l'ensemble des essais nécessaires à la mise en service industriel des machines a de nouveau été réalisé sur chacun des trois groupes, après les modifications énoncées ci-dessus. Les conditions hydrologiques n'autorisaient qu'un fonctionnement à deux groupes, avec en particulier un débit maximal de $10 \mathrm{~m}^{3} / \mathrm{s}$ et un niveau du réservoir amont inférieur d'environ $4,5 \mathrm{~m}$ à la cote de retenue normale. En conséquence, des essais complémentaires restent à faire avec trois groupes fonctionnant simultanément sous toutes les chutes, en particulier en pompe avec la plus forte hauteur de refoulement.

\section{IV $\square$ PERSPECTIVES D'AVENIR}

La mise en œuvre de pompes-turbines Deriaz à Naussac 2, proposée par Somival à I'EPALA, permettait de respecter les exigences d'adaptation aux conditions hydrologiques du site, tout en conservant à celui-ci sa beauté naturelle. Ce souci d'intégration à l'environnement était par ailleurs une donnée forte du projet, imposée par le Maître d'ouvrage et par le Maître d'œuvre.

D'autres sites analogues à celui de Naussac pourraient sans doute être équipés en France et à l'étranger. Les transferts d'eau, du même type que celui réalisé à Naussac, peuvent être envisagés chaque fois que doit être résolu un problème de soutien d'étiage ou éventuellement un problème d'inondation. L'utilisation de pompes-turbines Deriaz est dans ce cas parfaitement adaptée. Mais ce type de machines peut également s'intégrer dans tout projet de production d'énergie où il y a nécessité de fonctionner au fil de l'eau. D'ailleurs un ancien projet d'aménagement hydroélectrique dans les Alpes françaises, aujourd'hui abandonné pour des raisons écologiques, comportait un équipement intégrant des turbines Deriaz.

Aujourd'hui, l'aménagement de Naussac-Langogne constitue une vitrine française pour la démonstration de la faisabilité de projets à caractéristiques adaptées à l'environnement. L'ensemble des intervenants est prêt à accueillir à NAUSSAC les éventuels investisseurs français ou étrangers qui seraient intéressés par l'utilisation de pompes-turbines Deriaz et qui souhaiteraient en observer grandeur nature toutes les qualités de fonctionnement et de parfaite intégration au site. 